\title{
Vulnérabilité des aires protégées du Bénin à l'invasion de Ageratum conyzoides L. (Asteraceae) en rapport avec les changements climatiques
}

\author{
A. K. G. Djotan, BSc. \\ A. K. N. Aoudji, Dr. \\ Laboratoire des Sciences Forestières (LSF), Faculté des Sciences \\ Agronomiques (FSA), Université d'Abomey-Calavi (UAC), \\ Cotonou, République du Bénin \\ G. C. R. Gbaguidi, Ir. \\ G. S. Akouehou, Dr. \\ Centre d'Etudes, de Recherches et de Formation Forestières (CERF), \\ Ministère du Cadre de Vie et du Développement Durable (MCVDD), \\ Cotonou, République du Bénin \\ J. C. Ganglo, Professeur. \\ Laboratoire des Sciences Forestières (LSF), Faculté des Sciences \\ Agronomiques (FSA), Université d'Abomey-Calavi (UAC), \\ Calavi Bénin, République du Bénin
}

Doi: 10.19044/esj.2018.v14n33p313 URL:http://dx.doi.org/10.19044/esj.2018.v14n33p313

\begin{abstract}
Fighting against invasive species is a great challenge that requires knowledge about their potential distribution areas. Ageratum conyzoides is an invasive species in several African countries including Benin. In order to analyze the vulnerability of protected areas of Benin to the invasion of this species, and to guide managers of these areas to fight against invasions, this study used MaxEnt to model the ecological niche of the species under the current and future climates, horizon 2055 RCP 4.5 and RCP 8.5 scenarios. Environmental data and presence data were gathered respectively from the data bases of AfriClim and GBIF. From the current to the future climates, the potentially suitable areas for the distribution of the species shift from the center to the south of Benin. So, about $75 \%, 51 \%$ and $65 \%$ of Benin's total area are respectively suitable under present climate, and future's ones (RCP4.5 and RCP8.5). Only about $16 \%$ and $43 \%$ of this area are absolutely unsuitable and absolutely suitable to the species. As for protected areas, about $29 \%$ and $25 \%$ of their total area are respectively not vulnerable, and vulnerable to Ageratum conyzoides. Present climate, and future's ones
\end{abstract}


(RCP4.5, and RCP8.5) held respectively about 62\%, 28\%, and $48 \%$ of protected areas total superficies vulnerable to Ageratum conyzoides.

Keywords: Ageratum conyzoides, Invasive alien species, Vulnerability of protected areas, Ecological Modelling

\section{Résumé}

La lutte contre les espèces envahissantes est un grand défi qui nécessite des connaissances sur leurs zones de distribution potentielle. Ageratum conyzoides est une espèce envahissante dans plusieurs pays d'Afrique dont le Bénin. Dans le but d'analyser la vulnérabilité des aires protégées du Bénin à l'envahissement de cette espèce, et pour guider les gestionnaires de ces aires à lutter contre son invasion, la présente étude a utilisé MaxEnt pour modéliser la niche écologique de l'espèce sous les climats actuel et futurs, horizon 2055 des scénarii RCP 4.5 et RCP 8.5. Les données environnementales et les données de présence de l'espèce ont été obtenues respectivement des bases de données de AfriClim et de GBIF. Du climat actuel aux climats futurs, les aires potentiellement favorables à la distribution de l'espèce se déplacent du centre vers le Sud du pays. Ainsi, environ $75 \%, 51 \%$ et $65 \%$ de la superficie totale du Bénin sont respectivement favorables sous le climat actuel, sous les climats futurs RCP4.5 et RCP8.5. Seulement environ $16 \%$ et $43 \%$ de cette superficie sont respectivement absolument non favorables et absolument favorables à l'espèce. Quant aux aires protégées, environ 29\% et 25\% de leur superficie totale sont respectivement non vulnérables et vulnérables à l'envahissement de Ageratum conyzoides. Le climat actuel, les climats futurs RCP4.5 et 8.5 révélaient respectivement environ $62 \%, 28 \%$ et $48 \%$ de la superficie totale des aires protégées vulnérables à l'envahissement de Ageratum conyzoides.

Mots clés: Ageratum conyzoides, Espèces envahissantes, Vulnérabilité des aires protégées, Modélisation Ecologique

\section{Introduction}

La Convention des Nations Unies sur la Diversité Biologique définit une espèce envahissante comme une espèce qui est introduite intentionnellement ou accidentellement hors de son aire de répartition naturelle et qui cause de nombreux changements au sein des écosystèmes locaux, artificiels ou naturels. UNCBD (1992) reconnaît clairement que l'invasion est la deuxième plus grande menace qui pèse sur la biodiversité, et préconise donc que chaque partie contractante fasse de grands efforts pour prévenir et éradiquer toute introduction hasardeuse d'espèces dans les 
écosystèmes. L'ampleur du problème est très grande jusqu'au point où l'IUCN a mis au point des guides de prévention des pertes de la biodiversité liées aux invasions biologiques pour aider les pays, les agences de conservation, et les particuliers à réduire les menaces qui pèsent sur la biodiversité (ISSG, 2005). L'espèce envahissante, quelle que soit son importance sur d'autres plans surtout le plan médicinal (Okunade, 2002 ; Moura et al., 2005) constitue donc une menace potentielle que les gestionnaires des ressources naturelles et les conservateurs doivent combattre à bras le corps. Leurs actions néfastes sur l'écologie (Mack et al., 2000 ; Pimentel et al., 2005 ; Arker et Zuberi, 2009 ; Fandohan et al., 2015) peuvent s'étendre jusqu'aux aires de conservation de la biodiversité locale dont les aires protégées (Fandohan et al., 2015). La lutte contre ces espèces envahissantes nécessite la connaissance sur leurs zones de distribution potentielle aussi bien dans le climat actuel que dans le climat futur (Foxcroft et al., 2011 ; Taylor et Kumar 2013 ; Fandohan et al., 2015). La distribution d'une espèce est le résultat des multiples interactions entre plusieurs facteurs (Soberon, 2007) et certains de ces facteurs peuvent être utilisés pour modéliser les aires de distribution potentielle des espèces végétales ou animales d'intérêt (Philips et al., 2006; Pearson et al., 2007). Ageratum conyzoides L. est une espèce envahissante de certaines régions d'Asie, d'Afrique de l'Ouest et d'Amérique du Sud (Saunders et Stanley, 1999 ; Akter et Zuberi, 2009). Cette espèce remplit certaines conditions dont la facilité de dissémination et la spontanéité de germination sans préférence d'un type particulier de sol (Okunade, 2002) pour être une espèce envahissante au Bénin. La présente étude a pour objectif de modéliser la niche écologique de cette plante afin d'analyser la vulnérabilité des aires protégées du Bénin à son envahissement.

\section{Matériel et méthodes}

\section{Description de l'espèce}

Ageratum conyzoides $\mathrm{L}$. est une plante herbacée de la famille des Asteraceae commune á l'Afrique de l'Ouest et à certaines parties de l'Asie et de l'Amérique du Sud. Communément appelée herbe-à-bouc, elle est une petite plante dressée, poilue et plus ou moins branchue. Sa tige est robuste, souvent teintée de rouge et hérissée de poils. Les feuilles sont molles. Elles sont opposées de part et d'autre de la tige. Elles sont longuement pétiolées. Le limbe est couvert de poils sur les deux faces. Le bord des feuilles est régulièrement denté. Les nervures sont bien marquées. Les fleurs sont groupées en petites têtes, d'abord de couleur bleu-violacé pâle, puis blanches. Ses têtes sont associées en bouquets terminaux compacts. Le fruit contient une seule graine restant enfermée. A maturité, il est noir et surmonté d'une 
couronne d'écailles. La plante est une espèce annuelle ne dépassant pas généralement $1 \mathrm{~m}$ de hauteur. Elle se multiplie uniquement par graines et ses fruits sont transportés par le vent et par l'eau. Les fruits sont des akènes avec des graines photoblastiques tombant souvent après 12 mois. Les graines sont capables de germer immédiatement après leur dissémination. Elle n'a pas de préférence pour un sol en particulier, mais elle a suffisamment besoin d'humidité et de lumière pour se développer (Okunade, 2002). La figure 1 présente Ageratum conyzoides en floraison.



Figure 1. Ageratum conyzoides L. (Asteraceae) en floraison

\section{Milieu d'étude}

La République du Bénin est la zone d'intérêt pour la présente étude. Elle est un pays francophone situé entre les latitudes $6^{\circ} 30^{\prime}$ et $12^{\circ} 30^{\prime}$ Nord et la longitude $1^{\circ}$ et $3^{\circ} 40^{\prime}$ Est avec une superficie de $114.763 \mathrm{~km}^{2}$ (Ayihouenou et al., 2016). Sur le plan de l'environnement physique, le Bénin a un relief peu accidenté, marqué par cinq principaux ensembles géomorphologiques, à savoir : la plaine côtière sablonneuse, les plateaux sédimentaires du Continental Terminal, la pénéplaine cristalline, la chaîne de l'Atacora et la plaine de Gourma (DGE, 2011). Adomou et al. (2010) y avaient distingué dix phytodistricts en fonction des caractéristiques floristique et écologique dominantes. La figure 2 présente la zone d'étude. 


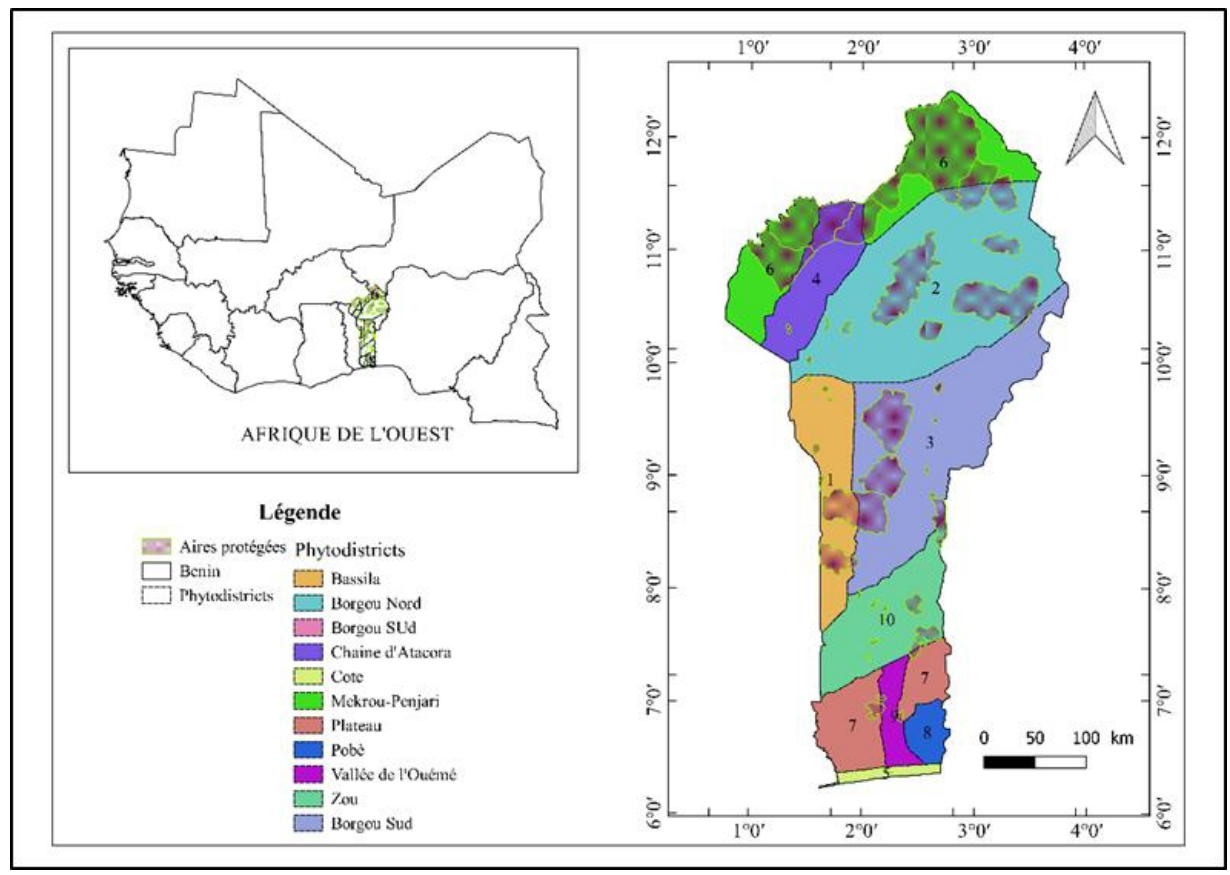

Figure 2: Carte de situation de la zone d'étude

\section{Données utilisées pour la modélisation}

La modélisation de niche écologique requiert des données environnementales et des données de présences. Dans le cadre de la réalisation de cette étude, les données environnementales considérées sont les variables bioclimatiques dont la pluviométrie, la température et leurs dérivés, disponibles sur le site d'AfriClim (Platts et al., 2015). Il existe plusieurs sites de téléchargement de ces variables, surtout à l'échelle globale mais ces derniers n'offrent pas autant de confidence pour les simulations climatiques, lesquels problèmes ne sont pas de grande ampleur lorsque l'on utilise les données de AfriClim (Platts et al., 2015) pour faire des études de modélisation de niches écologiques en Afrique.

Ainsi, les variables destinées à faire la modélisation des niches écologiques dans le climat actuel, correspondant au climato 1950-2000 (Hijmans et al., 2005), y ont été téléchargées suivant le lien https://webfiles.york.ac.uk/KITE/AfriClim/GeoTIFF_150s/baseline_worldcli $\underline{\mathrm{m} /}$. De plus, le téléchargement des variables du futur, correspondant à l'horizon 2055 sur la base de deux scénarii, RCP 4.5 et RCP 8.5 y ont été téléchargées pour les projections dans le futur, suivant le lien https://webfiles.york.ac.uk/KITE/AfriClim/GeoTIFF_150s/africlim_ensembl e_v3_worldclim/ (Platts et al., 2015). Les RCP sont des modèles climatiques basés sur les émissions et les politiques de protection de l'environnement, donnant des idées sur une probable évolution du climat (Stocker et al., 2013). 
Le RCP 4.5 et le RCP 8.5 font parties des scénarii les plus réalistes (Meinshausen et al., 2011). D'après leurs différentes prévisions, le RCP 4.5 est optimiste et le RCP 8.5 est pessimiste (Meinshausen et al, 2011). Le RCP 4.5 prévoit une stabilisation du forçage radiatif après 2100 sans dépassement de la valeur cible qui est de 4,5 W.m² (Clarke et al., 2007; Wise et al., 2009) alors que le RCP 8.5 quant à lui prévoit une augmentation sans fin des gaz à effet de serres dans le temps, conduisant ainsi à la plus forte concentration de ces gaz dans l'atmosphère (Riahi et al. 2007). Pour chacun de ces scénarii, le modèle moyen d'ensemble a été utilisé.

En ce qui concerne les données de présence, il s'agissait des coordonnées géographiques des points où l'espèce a été retrouvée ou bien présente. Ces données étaient obtenues sur le site de GBIF (Global Biodiversity Information Facility) suivant le lien https://doi.org/10.15468/dl.2ombkq. Ces données utilisées pour la modélisation sont accessible à tout moment suivant le lien http://www.gbif.org/occurrence/download/0001383-171219132708484. Ces points de présence couvraient aussi bien le Bénin que d'autres pays de la sousrégion afin de rendre le modèle plus prédictif (Fitzpatrick et Hargrove, 2009 ; Acevedo et al., 2012). La distribution des points de présence de l'espèce est présentée sur la figure 5.

\section{Techniques de modélisation}

L'algorithme de modélisation de niche écologique par l'approche d'entropie maximale (MaxEnt) (Philips et al., 2006; Pearson et al., 2007) a été utilisé dans cette étude pour modéliser les aires potentiellement favorables à la distribution de Ageratum conyzoides. En dehors de la définition aléatoire de $25 \%$ des points de présence de l'espèce pour tester le modèle et sortir une valeur du critère de validation Area «Under the Curve »-AUC- (Elith et al., 2006), tous les autres paramètres de MaxEnt étaient laissés à leurs valeurs par défaut pour tourner le modèle (Philips et Dudik, 2008) à des fins de sélection des variables qui influencent le plus la distribution de l'espèce. ENMTools a été utilisé pour calculer les corrélations entre les biovariables environnementales (Warren et al., 2010) utilisées à l'échelle de la zone de mobilité définie pour l'exécution du modèle. Deux biovariables environnementales étaient considérées comme étant fortement corrélées lorsque la valeur de la corrélation est au moins égale à 0,80 (Elith et al., 2011). La table des corrélations a été utilisée lors de l'élimination des variables afin de ne pas éliminer simultanément deux ou trois variables fortement corrélées au risque de perdre toutes les informations que portent ces dernières et qui pourraient ne pas Figurer dans celles apportées par d'autres. C'est ainsi que la table des contributions des biovariables et la table de Jackknife sur le gain des données d'entrainement ont été prises en compte pour sélectionner les cinq 
variables les plus à même capables d'expliquer la distribution de l'espèce. Les cinq variables retenues ont ensuite été utilisées pour tourner le modèle en mode «bootstap » sur 10 répétitions et les valeurs moyennes des paramètres de décision ont été utilisées. Il s’agit du modèle logistique de distribution de l'espèce, du seuil de décision qui exclut $10 \%$ des points de présence des aires favorables ( 10 percentile training presence »), du critère de validation AUC («Area Under the Curve ») et de sa déviation standard. Le seuil de décision qui a été retenu est celui-là qui minimisait le taux d'omission sur le test du modèle (erreur d'omission) et le taux d'omission sur les points de présence de l'espèce (erreur de commission), tout en offrant une valeur raisonnable de probabilité logistique de distribution.

\section{Techniques d'utilisation des résultats de MaxEnt}

Les logiciels de SIG et de Cartographie ont été utilisés pour traiter et présenter les résultats issus de MaxEnt à notre convenance conformément à l'objectif de l'étude. En effet, les cartes des aires favorables à l'espèce sont présentées d'une part sans classification et d'autre part après classification. La classification a été faite sur la base de la valeur du seuil retenu. Les aires où la probabilité logistique de distribution est inférieure au seuil sont définies de non favorables alors que les aires où cette valeur est supérieure au seuil sont définies comme aires favorables. La couche du réseau d'aires protégées a été superposée aux aires favorables à la distribution de l'espèce. Les portions des aires protégées qui appartiennent simultanément aux domaines favorables à l'espèce envahissante quel que soit le scenario étaient classées comme les zones absolument vulnérables, correspondant aux zones absolument favorables à la plante; et celles qui n'appartiennent pas à ce domaine quel que soit le scenario étaient traitées de zones non vulnérables, correspondant aux zones pas du tout favorables à la plante. De plus, les zones vulnérables selon chaque scenario ont été identifiées sur les cartes et leurs superficies ont été calculées.

\section{Résultats}

\section{Validation du modèle}

La valeur moyenne du critère d'évaluation est de 0,959 \pm 0,009 (Figure 4). Ces valeurs montraient que le modèle est excellent et stable. Les résultats de la table des seuils indiquaient que la probabilité associée à l'hypothèse nulle est de 3.056E-42 et nous permet de rejeter cette hypothèse, cette hypothèse étant «Les points d'évaluation du modèle ne sont pas prédits mieux qu'une prédiction aléatoire ». De ce fait, nous concluons que le modèle prédit mieux qu'une prédiction aléatoire. Le seuil qui exclut $10 \%$ des points de présence de l'espèce, et qui a été retenu pour la classification donnait une valeur moyenne de 0,245 pour la probabilité logistique en dessous de laquelle la zone est 
classée comme non favorable à l'espèce. A ce seuil de classification étaient associées une erreur de commission de 0,100 et une erreur d'omission de 0,183 . Les cinq variables qui étaient retenues (Tableau 1 et Figure 3) sont les variables bioclimatiques 11,2, 3, 17 et 6 . La variable 11 est la température minimale du trimestre le plus froid en $\left({ }^{\circ} \mathrm{C} \times 10\right)$, la variable 2 est l'amplitude thermique journalière en $\left({ }^{\circ} \mathrm{C} \times 10\right)$, la variable 3 est l'isothermalité en $\left({ }^{\circ} \mathrm{C} \times 10\right)$, les bio 17 et 6 sont respectivement la pluviométrie du trimestre le plus sec en $(\mathrm{mm})$ et la température minimale du mois le plus froid en $\left({ }^{\circ} \mathrm{C} \times 10\right)$.

\begin{tabular}{lrr}
\hline \multicolumn{3}{l}{ Tableau1. Contribution des variables } \\
\hline Variable & Percent contribution & Permutation importance \\
\hline Bio11 & 27 & 18,2 \\
Bio2 & 25,9 & 34,2 \\
Bio3 & 24,1 & 23,2 \\
Bio17 & 17,1 & 14,1 \\
Bio6 & 5,9 & 10,3 \\
\hline Les valeurs son ten pourcentage & & \\
\hline
\end{tabular}

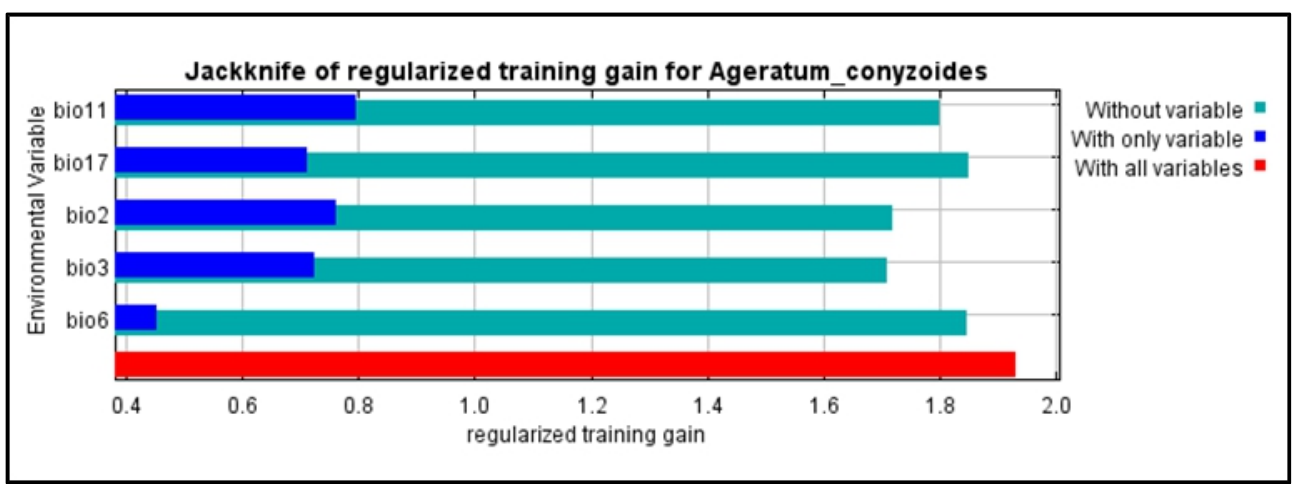

Figure 3: Jackknife du gain régularisé sur les données de calibration (Ageratum conyzoides) 


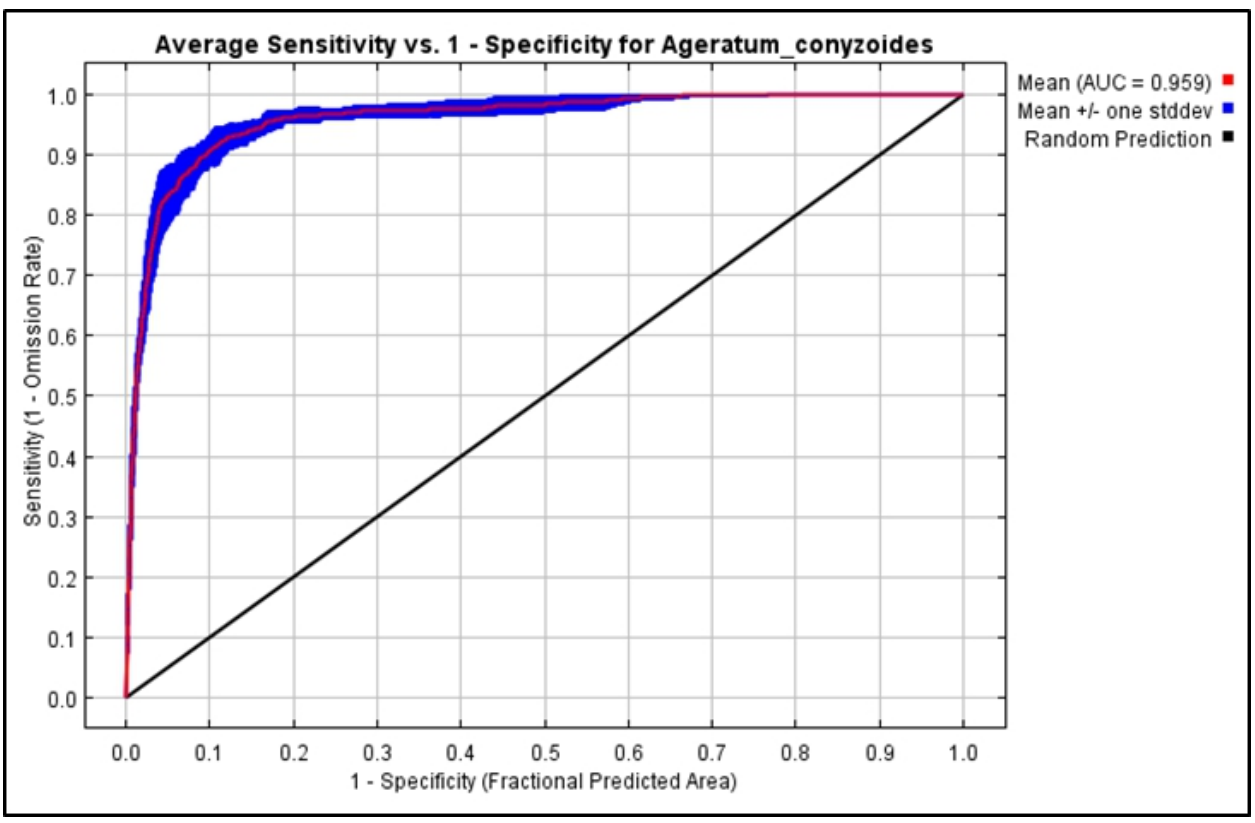

Figure 4: Courbe du receveur AUC

\section{Zones favorables à l'espèce selon les climats actuel et futurs}

Selon le climat actuel, les zones favorables à Ageratum conyzoides couvrent essentiellement des parties de certains phytodistricts dont le MékrouPenjari, la chaine de l'Atacora, la partie sud du Borgou Nord, la partie nord du Borgou Sud et une bande non moins importante dans la vallée de l'Ouémé. Les projections dans les climats futurs ont révélé un déplacement de l'enveloppe climatique de l'espèce. C'est ainsi que Ageratum conyzoides serait plus enclin à coloniser la partie sud du pays que la partie Nord. Cependant, notons que le phytodistrict du Mékrou-Penjari est resté très favorable à cette espèce. Le scénario RCP8.5 a révélé plus de zones favorables pour l'espèce que le scénario RCP4.5. Ainsi, selon le RCP8.5, la partie centrale du pays, partagée entre trois phytodistricts à savoir Borgou Sud, Borgou Nord et Bassila le sont également (Figure 6). Les Figures 5 et 6 présentent respectivement la distribution des points de présence de l'espèce et de ses zones favorables dans l'espace géographique de modélisation initiale, et la distribution des aires prédites favorables à l'espèce dans le climat actuel et les climats futurs, horizon 2055 au Bénin. Les points de présence qui sont en dehors du Bénin sur la figure 5 ne font qu'apporter plus de précision sur l'identification des zones potentiellement favorables à la distribution de Ageratum conyzoides. 


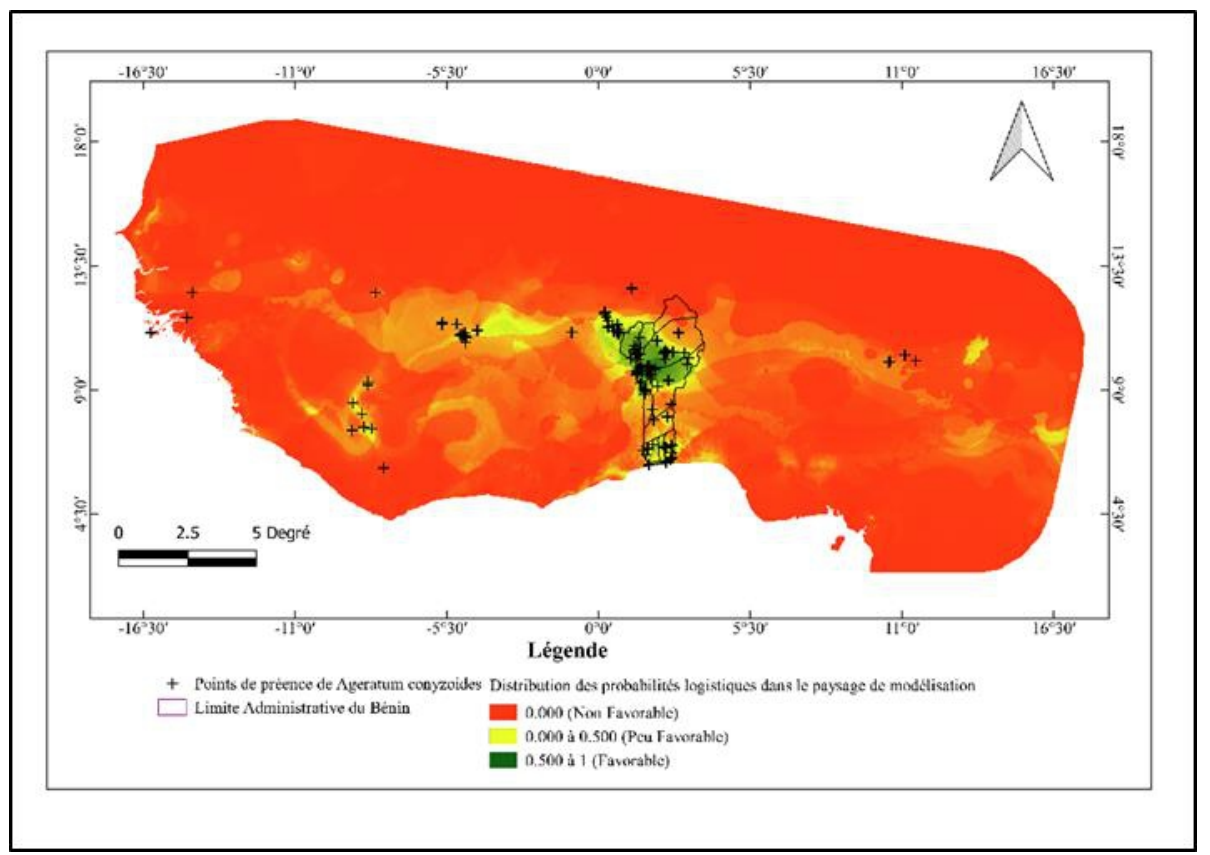

Figure 5 : Distribution des points de présence de l'espèce et de ses zones favorables dans l'espace géographique de modélisation initiale

Après la classification sur la base du seuil retenu, les zones favorables à l'espèce sont clairement visibles sur la figure 6 selon qu'il s'agisse du climat actuel ou des climats futurs. Une grande partie des aires protégées ainsi qu'une grande partie de tout le pays étaient révélées favorables à la plante. Le tableau 2 présente les statistiques sur les zones favorables à l'espèce sur le territoire national. La zone définie comme « absolument favorable » $(42,85 \%)$ est cette zone qui l'est quel que soit le climat ou le scenario. Par contre, «Pas du tout Favorable » correspond à la zone qui ne l'est pas quel que soit le climat ou le scenario utilisé $(16,12 \%)$. La prédiction sous le climat du présent montrait $74,84 \%$ de la superficie totale du territoire nationale comme favorable alors que les scenarii RCP4.5 et RCP8.5 en ont prédit respectivement $51,38 \%$ et $64,76 \%$ (Figure 7).

Tableau2. Aires favorables à l'espèce sur le territoire national

\begin{tabular}{lrrrrr}
\hline $\begin{array}{l}\text { Pcenario/Statut } \\
\text { Superficie }\left(\mathbf{K m}^{2}\right)\end{array}$ & $\begin{array}{r}\text { Favorable } \\
18612,00\end{array}$ & $\begin{array}{r}\text { Absolument } \\
\text { favorable } \\
49481,29\end{array}$ & $\begin{array}{r}\text { Favorable sous } \\
\text { climat actuel } \\
86407.79\end{array}$ & $\begin{array}{r}\text { Favorable sous } \\
\text { RCP45 }\end{array}$ & $\begin{array}{r}\text { Favorable sous } \\
\text { RCP85 }\end{array}$ \\
\hline $\begin{array}{l}\text { Pourcentage de la } \\
\text { superficie totale } \\
\text { du Bénin (\%) }\end{array}$ & & & & & \\
\hline
\end{tabular}




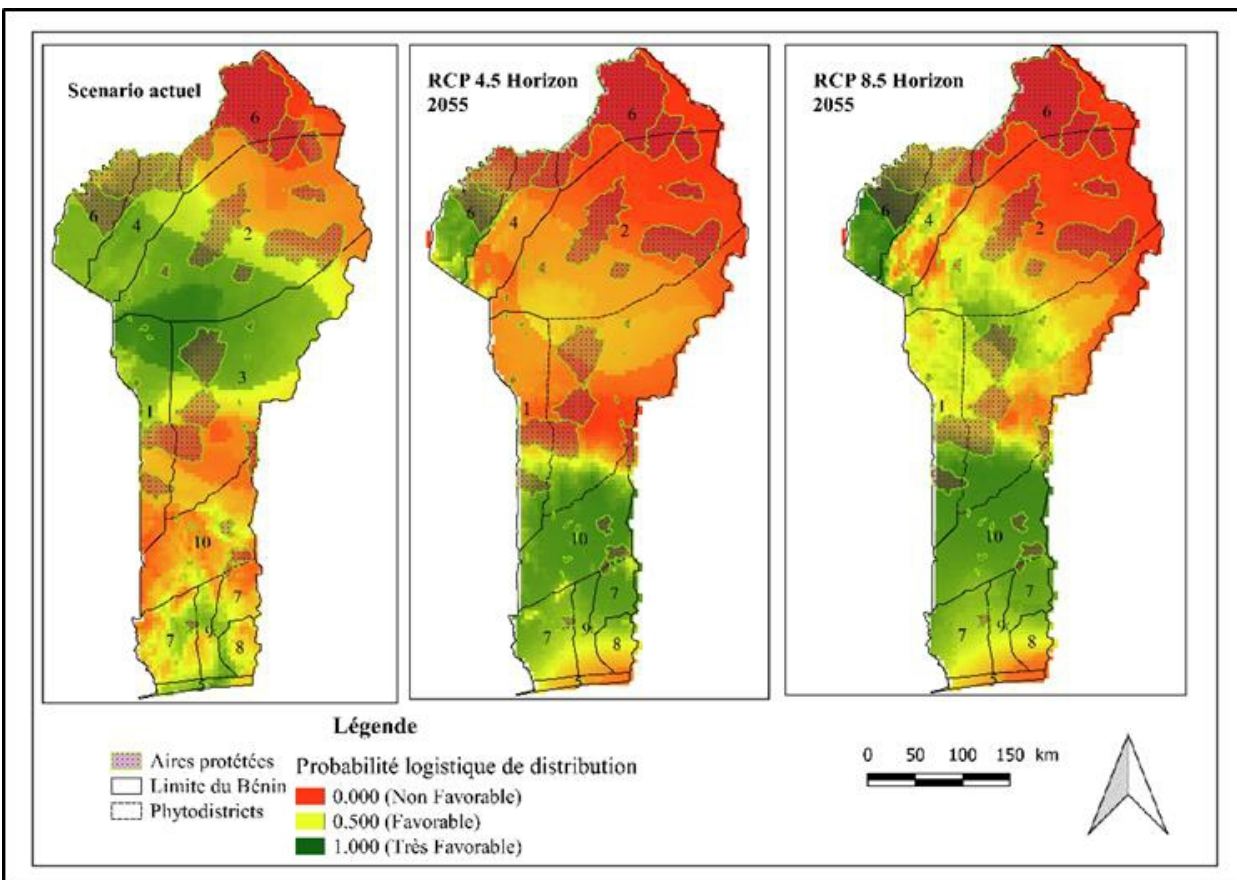

Figure 6: Distribution des aires prédites favorables à l'espèce dans le climat actuel et les climats futurs, horizon 2055

\section{Vulnérabilité des aires protégées à l'espèce dans les climats actuel et futurs, horizon 2055}

Ageratum conyzoides est une espèce envahissante et pourrait constituer une menace pour nos aires protégées. Les aires protégées qui ne sont pas du tout dans le domaine des zones favorables à cette espèce ne sont pas du tout vulnérables. Par contre, celles qui sont dans les zones absolument favorables à l'espèce en sont absolument vulnérables. La figure 8 présente la distribution de ces zones de vulnérabilité, et le tableau 3 présente les statistiques concernant ces zones de vulnérabilité.

Tableau 3. Vulnérabilité des aires protégées à l'espèce

\begin{tabular}{lrrrrr}
\hline Scenario/Statut & $\begin{array}{r}\text { Aucune } \\
\text { vulnérabilité }\end{array}$ & $\begin{array}{r}\text { Vulnérabilité } \\
\text { totale }\end{array}$ & $\begin{array}{r}\text { Vulnérable } \\
\text { sous Climat } \\
\text { actuel }\end{array}$ & $\begin{array}{r}\text { Vulnérable } \\
\text { sous RCP }\end{array}$ & $\begin{array}{r}\text { Vulnérable } \\
\text { sous RCP }\end{array}$ \\
Superficie $\left(\mathbf{K m}^{\mathbf{2}}\right)$ & 7892,13 & 7055,21 & 17311,83 & 7828,69 & 13290,19 \\
\hline $\begin{array}{l}\text { Pourcentage de la } \\
\text { superficie totale des AP } \\
\text { (en \%) }\end{array}$ & 28,45 & 25,43 & 62,41 & 28,22 & 47,91 \\
\hline
\end{tabular}

$28,45 \%$ de la superficie totale des aires protégées est exempt de l'invasion éventuelle de Ageratum conyzoides. Cette zone couvre en partie les aires protégées de Toui-Kilibo, Tchaourou, Wari-Maro, Mékrou, Sota, Goungoun, la zone cynégétique de la Djona, les parcs nationaux de la Penjari 
et du W (Figure 8). Quant aux aires protégées absolument vulnérables, leurs superficies concernées représentent $25,43 \%$ de la superficie totale des aires protégées et comprend en partie la zone cynégétique de la Penjari, le parc de la Penjari, les forêts classées de Mékrou, Alibori supérieur, Kouandé, Béléfoungou, Ouémé supérieur, Monts Kouffé, Agoua, DOgo-Kétou, Ouémé Boukou, Savalou, Logozohè, Lama, Agrimè, Atchérigbé, Djigbé, Ouèdo et Pahou (Figure 8). Le climat actuel, les climats futurs RCP 4.5 et RCP 8.5 ont prédit respectivement $62,41 \%, 28,22 \%$ et $47,91 \%$ de la superficie total des aires protégées comme vulnérables à l'invasion éventuelle de Ageratum conyzoides (Figure 7).

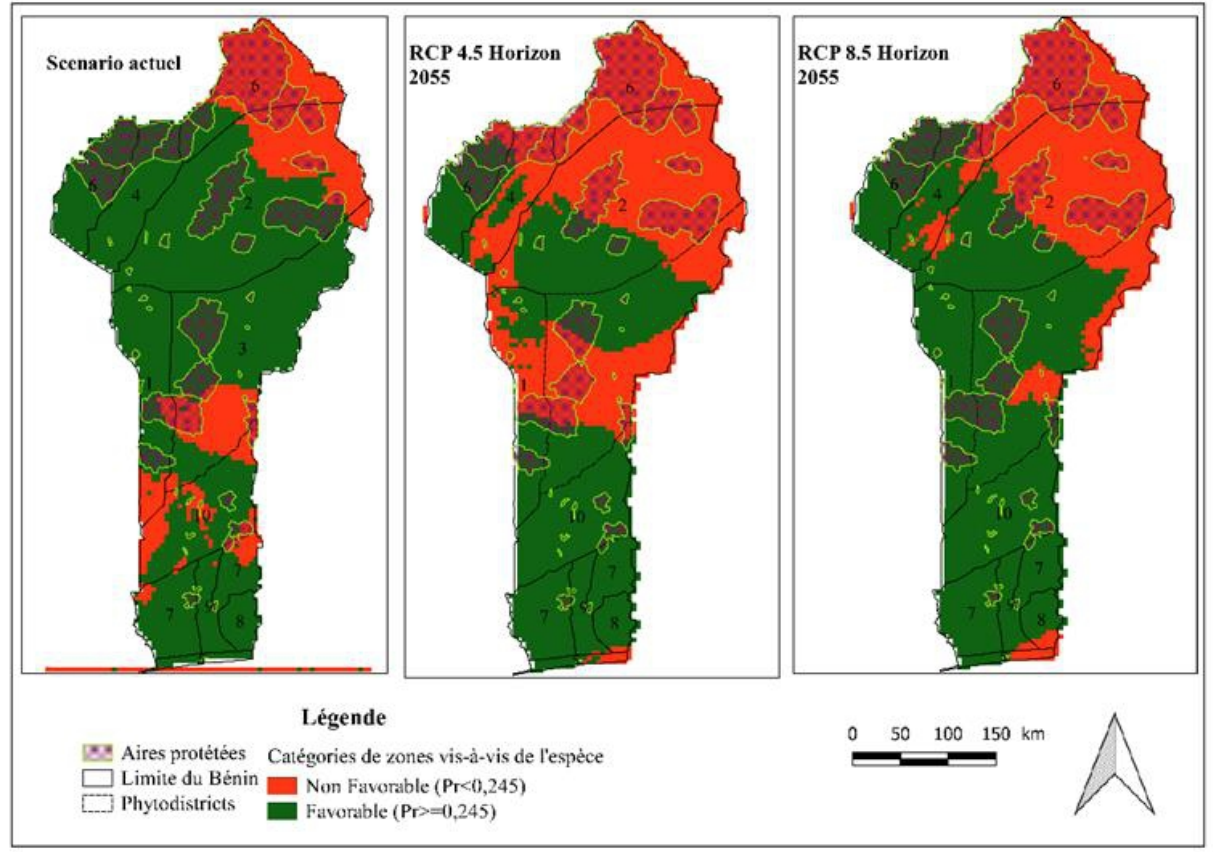

Figure 7: Distribution après classification des zones favorables à l'espèce dans le climat actuel et les climats futurs, horizon 2055

\section{Discussion}

Notre étude a ressorti les variables qui contribuent les plus à la distribution de l'espèce dont la niche écologique a été modélisée et superposée aux couches du réseau des aires protégées du Bénin. Ageratum conyzoides est une plante annuelle herbacée, et donc les modèles de distribution incluant le type de sol ou aussi la composition organique et ou chimique du sol pourrait être meilleurs (Taylor et Kumar 2013). De plus, Pearson et Dawson (2003) ont recommandé d'utiliser la variable sol pour estimer les habitats favorables à la distribution d'une espèce lorsque la modélisation est faite à une échelle en dessous de $2000 \mathrm{~km}$. Cependant, cette variable n'a pas été prise en compte dans la présente modélisation puisque d'après Okunade (2002), l'espèce aurait 
une facilité de dissémination et une spontanéité de germination sans préférence d'un type particulier de sol. Ceci nous maintien à l'écart des doutes liés au fait de supposer que la variable sol ne change pas dans le climat futur, si cette dernière était utilisée (Allen et al., 2011) car c'est toujours un défi majeur de définir les caractéristiques du sol dans un climat futur (Wixon et Balser, 2009). Okunade (2002) mentionnait que Ageratum conyzoides a suffisamment besoin de lumière et d'humidité. Cette information est conforme à celle qui découle des variables les plus importantes que le modèle a révélées.

Le seuil qui exclut $10 \%$ des points de présence de l'espèce a été utilisé à l'instar de Fandohan et al. (2015) pour classifier les résultats obtenus à partir de MaxEnt. L'utilisation des seuils est parfois subjective mais doit essentiellement dépendre des objectifs de l'étude ainsi que les applications éventuelles (Peterson et al., 2008). Dans notre cas, ce seuil minimise les erreurs de commission et d'omission et est donc idéal, permettant d'éviter la surestimation ou la sous-estimation des aires potentiellement favorable à une espèce envahissante. Ganglo et al. (2017) ont utilisé deux différents seuils pour appréhender la différence significative entre les classifications avec application directe à la conservation de Dialium guineense Wild. (Fabaceae) en s'inscrivant dans la logique de Peterson et al. (2008).

Nos résultats ont aussi montré que les aires favorables à la distribution de Ageratum conyzoides ne sont pas restées les mêmes du climat actuel aux climats futurs. Ceci est conforme aux prédictions de plusieurs auteurs dont McClean et al. (2005) et Alig (2011) qui stipulaient que les aires favorables des espèces de plante sont en perpétuel déplacement et que d'autres déplacements sont attendus en raison des changements climatiques. Beaucoup de ces déplacements peuvent porter sur des espèces envahissantes dont Ageratum conyzoides.

La modélisation des aires potentielles de distribution de cette espèce ainsi que les projections dans le futur, horizon 2055 ont montré qu'elle constitue une menace pour nos aires protégées. Fandohan et al. (2015) avaient trouvé des résultats similaires après avoir modélisé dans les climats actuel et futur, les distributions de Chromolaena odorata (L.) R.M.King \& H.Rob. (Asteraceae) qui est elle aussi une espèce envahissante. Ces genres d'études confirment les suppositions de Pearson et al. (2007) et de Peterson et al. (2011) et qui disaient que l'on peut tirer énormément d'avantages à partir des outils de modélisation pour résoudre certains des problèmes que rencontre la gestion des ressources naturelles dans le monde. 


\section{Conclusion et perspectives}

La modélisation de niche effectuée a permis de ressortir les aires protégées qui seraient éventuellement soumises à une invasion biologique par Ageratum conyzoides. Les impacts du changement climatique ont aussi été évalués sur l'étendue de la distribution potentielle de l'espèce, permettant de prendre des mesures préventives. Les résultats obtenus constituent un guide de prévention des invasions biologiques. Il serait très utile de multiplier des études similaires au Bénin pour respecter les instructions données par l'énoncé de l'article 8 de la Convention des Nations Unies sur la Diversité Biologique. Il urge aux gestionnaires des parcs et des aires protégées concernés de rester attentifs pour observer les moindres indicateurs de l'invasion par Ageratum conyzoides. Les zones prédites actuellement favorables à cette espèce et appartenant aux domaines des aires protégées doivent impérativement faire objets de prospection afin de contrecarrer avec des mesures idoines l'envahissement par cette plante qui pourrait modifier totalement à la longue l'écologie du milieu.

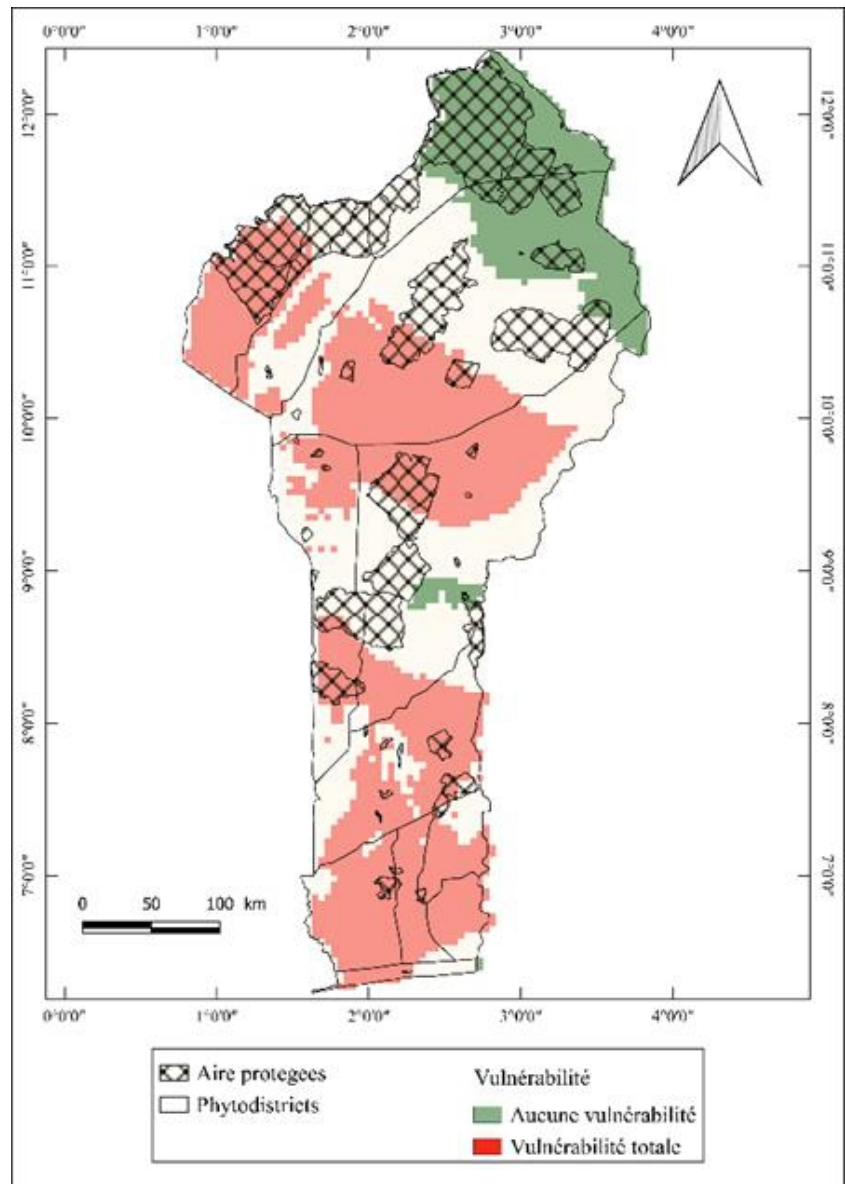

Figure 8: Distribution de la vulnérabilité des aires protégées à l'envahissement de Ageratum conyzoides 


\section{References:}

1. Acevedo, P., Jiménez-Valverde, A., Lobo, J. M., Real, R. (2012). Delimiting the geographical background in species distribution modelling. Journal of Biogeography, 39, 1383-1390.

2. Adomou, A. C., Sinsin, B., Akoégninou, A. A., \& Maesen, J. (2010). Plant species and ecosystems with high conservation priority in Benin. In Systematics and conservation of African plants. Proceedings of the 18th AETFAT Congress, Yaoundé, Cameroun, 26 February to 2 March 2007 (pp. 429-444). Royal Botanic Gardens.

3. Akter, A., Zuberi, M. I. (2009). Invasive alien species in Northern Bangladesh: identification, inventory and impacts. International journal of biodiversity and conservation, 1(5), 129-134.

4. Allen, D. E., Singh, B. P., Dalal, R. C. (2011). Soil health indicators under climate change: a review of current knowledge. Pages 25-45 in B. P. Singh, A. L. Cowie, and K. Y. Chan, editors. Soil health and climate change. Soil biology 29. Springer-Verlag, Berlin Heidelberg, Germany.

5. Alig, Ralph J., tech. coord. (2011). Effects of climate change on natural resources and communities: a compendium of briefing papers. Gen. Tech. Rep. PNWGTR-837. Portland, OR: U.S. Department of Agriculture, Forest Service, Pacific Northwest Research Station. 169 p.

6. Ayihouenou, E. B., Fandohan, A. B., Sodé, A. I., Gouwakinnou, N. G., Djossa, A. B. (2016). Biogéographie du néré (Parkia biglobosa (Jack.) R. Br. ex. Don.) sous les conditions environnementales actuelles et futures au Bénin. Bulletin de la Recherche Agronomique du Bénin, Numéro spécial Agronomie, Société, Environnement \& Sécurité Alimentaire. ISSN sur papier (on hard copy) : 1025-2355 et ISSN en ligne (on line) : 1840-7099.

7. Clarke, L., Edmonds, J., Jacoby, H., Pitcher, H., Reilly, J., Richels, R. (2007). Scenarios of Greenhouse Gas Emissions and Atmospheric Concentrations (Sub-report 2.1A of Synthesis and Assessment Product 2.1, US Climate Change Science Program and the Subcommittee on Global Change Research, Department of Energy, Office of Biological \& Environmental Research, Washington DC, 2007)

8. Direction Générale de l'Environnement. (2011). Deuxième Communication Nationale de la République du Bénin sur les Changements Climatiques. Cotonou: Ministère de l'Environnement, de l'Habitat et de l'Urbanisme.

9. Elith, J., Graham, C.H., Anderson, R.P., Dudık, M., Ferrier, S., Guisan, A., Hijmans, R.J., Huettmann, F., Leathwick, J.R., Lehmann, A., Li, 
J., Lohmann, L.G., Loiselle, B.A., Manion, G., Moritz, C., Nakamura, M., Nakazawa, Y., Overton, J.M., Peterson, A.T., Phillips, S.J., Richardson, K., Scachetti-Pereira, R., Schapire, R.E., Soberon, J., Williams, S., Wisz, M.S., Zimmermann, N.E. (2006). Novel methods improve prediction of species' distributions from occurrence data. Ecography 29: 129/151.

10. Elith, J., Kearney, M., Philips, S. (2011). The art of modeling rangeshifting species. Methods in Ecology and Evolution 1:330-342.

11. Fandohan, A.B., Oduor, A.M.O., Sodé, A.I., Wu, L., Cuni-Sanchez, A., Assédé, E., Gouwakinnou, G.N. (2015). Modeling vulnerability of protected areas to invasion by Chromolaena odorata under current and future climates. Ecosystem Health and Sustainability, 1(6):20.

12. Fitzpatrick, M.C., Hargrove, W.W. (2009). The projection of species distribution models and the problem of non-analog climate. Biodiversity Conservtion. 18: 2255-2261.

13. Foxcroft, L. C., Jaròsík, V., Pysek, P., Richardson, D. M., Rouget, M. (2011). Protected area boundaries as a natural filter of plant invasions from surrounding landscapes. Conservation Biology 25:400-405.

14. Ganglo, J.C., Djotan, G.K., Gbètoho, J.A., Kakpo, S.B., Aoudji, A.K.N., Koura, K., Tessi, D.R.Y. (2017). Ecological niche modeling and strategies for the conservation of Dialium guineense Willd. (Black velvet) in West Africa. International Journal of Biodiversity and Conservation. 9(12) : 373-388.

15. GBIF. (2017). GBIF Occurrence Download https:// doi.org/10.15468/dl.2ombkq. GBIF.org (24th December 2017)

16. Hijmans R.J., Cameron S.E., Parra J.L., Jones P.G., Jarvis A., (2005). Very high resolution interpolated climate surfaces for global land areas. International Journal of Climatology. 25:1965-1978.

17. ISSG (Invasive Species Specialist Group). (2005). Annex 2. Select alien invasive species affecting forests and forestry Annex 3. International and regional instruments and programmes. http://ww.issg.org.

18. Mack, R. N., Simberloff, D., Lonsdale, W. M., Evans, H., Clout, M., Bazzaz, F. A. (2000). Biotic invasions: causes, epidemiology, global consequences, and control. Ecological Applications 10:689-710.

19. Meinshausen, M., Smith, J., Calvin, K., Daniel, J. S., Kainuma, M. L. T., Lamarque, J-F., Matsumoto, K., Montzka, S. A., Raper, S. C. B., Riahi, K., Thomson, A., Velders, G. J. M., van Vuuren, D. P. P. (2011). "The RCP greenhouse gas concentrations and their extensions from 1765 to 2300 (open access)", Climatic Change, 109 (1-2): 213-241.

20. McClean, C. J., Lovett, J. C., Küper, W, Hannah, L., Sommer, J. H., Barthlott, W., Termansen, M., Smith, G. F., Tokumine, S., Taplin, J. 
R. D. (2005). African plant diversity and climate change. Annals of the Missouri Botanical Garden, 92(2) : 139-152.

21. Moura, A. C. A., Silva, E. L. F., Fraga, M. C. A., Wanderley, A. G., Afiatpour, P., Maia, M. B. S. (2005). Antiinflammatory and chronic toxicity study of the leaves of Ageratum conyzoides L. in rats. Phytomedicine, 12(1-2), 138-142.

22. Okunade, A. L. (2002). Ageratum conyzoides L. (Asteraceae). Fitoterapia, 73:1-16

23. Pearson, R. G., Dawson, T. P. (2003). Predicting the impacts of climate change on the distribution of species: Are bio climate envelope models useful? Global Ecology and Biogeography, 12:361-371.

24. Pearson, R. G., Christopher, J., Raxworthy, M. N., Peterson, A.T. (2007). Predicting species distributions from small numbers of occurrence records: a test case using cryptic geckos in Madagascar. Journal Biogeography, 34:102-117

25. Peterson, A. T., Papes, M., Soberon, J. (2008). Rethinking receiver operating characteristic analysis applications in ecological niche modeling. Ecological Modelling. 213:63-72.

26. Peterson, A. T., Soberón, J., Pearson, R. G., Anderson, R. P., MartínezMeyer, E., Nakamura, M., Araújo, M. B. (2011). Ecological Niches and Geographic Distributions. Princeton University Press, Princeton

27. Philips, S. J., Anderson, R. P., Schapire, R. E. (2006). Maximum entropy modelling of species geographic distributions. Ecological Modelling, 190:231-259

28. Philips, S. J., Dudik, M. (2008). Modeling of species distributions with Maxent. New extensions \& a comprehensive evaluation. Ecography, 31:61-175.

29. Pimentel, D., Zuniga, R., Morrison, D. (2005). Update on the environmental and economic costs associated with alien invasive species in the United States. Ecological Economics, 52:273-288.

30. Platts, P. J., Omeny, P. A., Marchant, R. (2015). AFRICLIM: highresolution climate projections for ecological applications in Africa. African Journal of ecology, (C) 2014 John Wiley \& Sons Ltd, African Journal of Ecoogy, 53:103-108.

31. Riahi, K., Gruebler, A., Nakicenovic, N. (2007). Scenarios of longterm socio-economic and environmental development under climate stabilization. Technol Forecast Soc Chang, 74:887-935, Special Issue: Greenhouse Gases - Integrated Assessment.

32. Saunders, K., Stanley, J. (1999). A Nanovirus-like DNA Component Associated with Yellow Vein Disease of Ageratum conyzoides: Evidence for Interfamilial Recombination between Plant DNA Viruses. Virology 264:142-152. 
33. Soberón, J. (2007). Grinnellian and Eltonian niches and geographic distributions of species. Ecology Letters, 10:1115-1123.

34. Taylor S., Kumar L., (2013). Potential distribution of an invasive species under climate change scenarios using CLIMEX and soil drainage: a case study of Lantana camara L. in Queensland, Australia. Journal of Environmental Management, 114:414-422.

35. Stocker, T. F., Qin, D., Plattner, G. K., Tignor, M., Allen, S. K., Boschung, J., ... \& Midgley, P. M. (2013). IPCC, 2013: Climate Change 2013: The Physical Science Basis. Contribution of Working Group I to the Fifth Assessment Report of the Intergovernmental Panel on Climate Change, $1535 \mathrm{pp}$.

36. UNCBD. (1992). Convention on biological diversity, Text and Annexes. The Interim Secretariat for the UNCBD, Geneva Executive Center.

37. Warren D. L., Glor R. E., Turelli M., (2010). ENMTools: a toolbox for comparative studies of environmental niche models. Ecography, 33: 607-611.

38. Wise, M. A., Calvin, K. V., Thomson, A. M., Clarke, L. E., BondLamberty, B., Sands, R. D., Smith, S. J., Janetos, A. C., Edmonds, J. A. (2009). The Implications of Limiting CO2 Concentrations for Agriculture, Land Use, Land-use Change Emissions and Bioenergy. Pacific Northwest National Laboratory Operated By Battelle For The United States Department Of Energy Under Contract DE-AC0576RL01830.

39. Wixon, D. L., Balser, T. C. (2009). Complexity, climate change and soil carbon: a systems approach to microbial temperature response. Systems Research Behavioral Science, 26:601-620. 